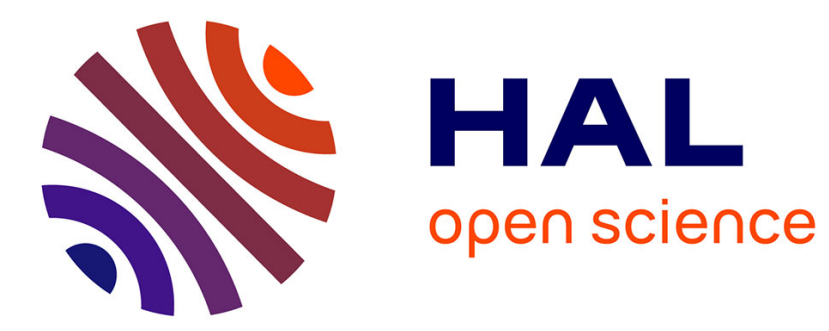

\title{
SPIKE, an automatic theorem prover - revisited
}

Sorin Stratulat

\section{To cite this version:}

Sorin Stratulat. SPIKE, an automatic theorem prover - revisited. SYNASC2020 22nd International Symposium on Symbolic and Numeric Algorithms for Scientific Computing, Sep 2020, Timisoara, Romania. pp.93-96. hal-02965319v2

\section{HAL Id: hal-02965319 \\ https://hal.science/hal-02965319v2}

Submitted on 8 Dec 2020

HAL is a multi-disciplinary open access archive for the deposit and dissemination of scientific research documents, whether they are published or not. The documents may come from teaching and research institutions in France or abroad, or from public or private research centers.
L'archive ouverte pluridisciplinaire HAL, est destinée au dépôt et à la diffusion de documents scientifiques de niveau recherche, publiés ou non, émanant des établissements d'enseignement et de recherche français ou étrangers, des laboratoires publics ou privés. 


\title{
SPIKE, an automatic theorem prover - revisited
}

\author{
Sorin Stratulat \\ Université de Lorraine, CNRS, LORIA \\ F-57000 Metz, France \\ Email: sorin.stratulat@loria.fr
}

\begin{abstract}
SPIKE, an induction-based theorem prover built to reason on conditional theories with equality, is one of the few formal tools able to perform automatically mutual and lazy induction. Designed at the beginning of 1990s, it has been successfully used in many non-trivial applications and served as a prototype for different proof experiments and extensions. The first paper introducing SPIKE is [14], published shortly after the tool was created. The goal of this paper is to highlight and bring together in one spot the major changes supported by SPIKE since then.
\end{abstract}

1) Introduction: Historically, SPIKE was built during a period when several formula-based Noetherian induction methods issued from Musser's completion-based inductionless induction (or proof-by-consistency) technique [33] have been designed. Some of them have been implemented into theorem provers, for example, RRL [30] integrated the test-set induction method [29], and Focus [20] a generalization of the termrewriting induction [21] for conditional theories. Inspired by the rewriting techniques previously tested with the ORME system [32], SPIKE [14] implemented a different induction method [15], [31] that combines features from explicit induction and inductive completion techniques.

As time went by, SPIKE was continuously considered among the 'active' automatic induction-based provers; it mainly served as a prototype for testing several extensions of conditional theories and induction-based reasoning techniques that led to many successful proof experiments on non-trivial applications. The goal of the paper is to highlight the major changes supported by SPIKE since the publication of [14], which gave rise to its current version. The source code, examples of specification files, and papers related to different applications and extensions are available online [43].

In the rest of the paper, we set the theoretical backgrounds of the reasoning by induction on equational clauses, then we introduce the inference system of SPIKE and the layout of a standard specification file. In the end, we show how to prove conjectures and interact with the tool.

\section{2) The SPIKE Prover:}

a) The 'reasoning by induction' setting: SPIKE implements an instance of the Noetherian induction principle applied on a Noetherian poset of equational clauses (or just clauses). Compared to other instances adapted for first-order reasoning, several clauses can be simultaneously tested to verify whether they are consequences of a given set of axioms $A x$ written as conditional equalities. SPIKE can reason on sorted and constructor-based specifications that should satisfy some properties, like the ground convergence and (strongly sufficient) completeness. These constraints guarantee the existence of the initial model for $A x$. Formally, assuming that
$\mathcal{M}$ is the initial model of $A x$, we denote by $\Phi \models \mathcal{M} \phi$ the fact that the clause $\phi$ is an initial $\mathcal{M}$-consequence (or just consequence) of a set of conditional equalities $\Phi . \phi$ is initially $\mathcal{M}$-valid (or just valid) and is denoted by $\models_{\mathcal{M}} \phi$ iff it is a consequence of $A x$. In general, given $(\mathcal{E}, \leq)$ a non-empty poset of formulas that is wellfounded (or Noetherian), i.e., any strictly decreasing sequence of formulas from $\mathcal{E}$ is finite, the formula-based instance [38] of the Noetherian induction principle states that if $\{\gamma \mid \gamma \in \mathcal{E}, \gamma<\delta\} \mid=_{\mathcal{M}} \delta$, for any formula $\delta \in \mathcal{E}$, then for all $\rho \in \mathcal{E},=_{\mathcal{M}} \rho$. Put it in a simpler way for our logical context, during the proof of a clause, smaller clauses can be used in terms of induction hypotheses (IHs). This makes it a natural choice for reasoning on proofs requiring lazy induction, when the IHs are generated by need, and mutual induction, when different clauses need each other to cross fertilize for making the induction reasoning successful. The induction proof method used by SPIKE, called implicit induction, is an application of this principle using reductive techniques as rewriting. It was firstly suggested in [31] and formally presented in [21]. The mutual induction feature helped SPIKE to prove the Gilbreath Card trick problem [28], firstly with 5 lemmas [16] then with only 2 lemmas [17], while other provers using different proof techniques succeeded with significantly more lemmas (see [17] for a comparison).

The ground convergence and completeness properties of a specification can be checked more easily, by using syntactic criteria, if the specification is many sorted and the set of function symbols is split into constructor and defined function symbols. SPIKE was initially designed to deal with free constructors such that there is no equality relation between any two different constructor symbols. Several extensions have been introduced in SPIKE since [14] in order to deal with: i) non-free constructors [13], ii) parameterized specifications [9], [10], iii) associative-commutative theories [6], iv) observational proofs [7], [18], and v) simultaneous check of the completeness and ground convergence properties of a specification [12]. Most of them led to distinct proof systems that are no longer maintained in spite of their theoretical and practical interests.

b) The inference system: In [14], the inference rules and the proof strategy implementing the implicit induction method were built-in. Each rule is a transition between pairs $(E, H)$, where $E$ are conjectures and $H$ are premises consisting of previously processed conjectures that do not have minimal counterexamples, i.e., minimal ground clauses that are not valid. By applying a rule, a conjecture from the current proof state is replaced by a potentially empty set of new conjectures, and may be added as a premise in order to participate to further inference steps. Proof derivations are built by successively applying inference rules starting from an initial state. They 
may finish with i) success, if they end with an empty set of conjectures, ii) error, if a counterexample is detected, and iii) failure, if none of the previous cases is encountered and no rule can be applied. A proof is a successful derivation that starts with an empty set of premises.

Later on, different proof needs led to hardcode into the system several variants of a same rule. More flexibility has been achieved with the addition of a strategy language [1] allowing to define new proof strategies by the user. It has been combined later with a methodology for building modular inference rules using contextual cover sets (CCSs) [36]. The core of the methodology is an abstract inference system made of two rules: ADDPREMISE and SIMPLIFY, defined as:

AddPREmise: $(E \cup\{\phi\}, H) \vdash_{A}(E \cup \Phi, H \cup\{\phi\})$,

if, for any counterexample (ctx.) $\phi \tau$ of $\phi$, it is a ctx. $\psi$ in

i) $E \cup \Phi$ such that $\psi<\phi \tau$, or

ii) $H$ such that $\psi \leq \phi \tau$.

SIMPlify: $(E \cup\{\phi\}, H) \vdash_{A}(E \cup \Phi, H)$,

if, for any ctx. $\phi \tau$ of $\phi$, there is a ctx. $\psi$ in

$E \cup \Phi \cup H$ such that $\psi \leq \phi \tau$.

Each inference rule replaces a conjecture $\phi$ with a potentially empty set of new conjectures $\Phi$. $\Phi$ is built in two steps as a CCS of $\phi$, thanks to the compositional properties of CCSs. Firstly, an intermediate CCS of the replaced conjecture is built, then for each intermediate clause another CCS is built and stored as new conjectures. The set of IHs allowed by a rule to be used when building a CCS is referred to as context. ADDPREMISE adds $\phi$ as a premise and SIMPLIFY allows bigger contexts. It has been shown in [36] that i) the abstract inference system is sound, i.e., one can conclude from a proof that its initial conjectures are valid, and ii) the inference rules define the biggest contexts compared to similar abstract rules proposed in the literature. For practical reasons, the abstract system was extended with a third rule, DeLETE, that is a particular case of SIMPLIFY when $\Phi$ is empty.

Any SPIKE inference rule instantiates one of the abstract rules by implementing its elementary CCSs, i.e. the CCSs that are not built using composition operations, by the means of reasoning modules. A reasoning module can produce a CCS with a particular reasoning technique using in terms of IHs clauses from the context defined by the instantiated abstract rule. The main reasoning techniques are based on rewriting, case analysis and variable instantiations.

We give as example the definition of a rewriting-based inference operation implemented as an instance of SIMPLIFY:

rewriting_rule $=$

simplify (id, [rewriting (rewrite, L|R|C, *) ] ) ;

In the first step, the identity reasoning module id builds $\{\phi\}$ as the intermediate CCS for $\phi$. The application of the rule succeeds if, in the second step, the rewriting module succeeds to rewrite once, due to the rewrite argument and at any position $(*)$, the only clause $\phi$ of $\{\phi\}$ with conditional rewrite rules from the lemmas (L), axioms (R) and current context (C). The resulting clause is the unique clause of $\Phi$.
Some of the reasoning techniques have been changed since [14]. For example, the technique for instantiating variables with elements of a test-set, based on the depth of the lhs of the axioms [17], was replaced by a narrowing technique involving only the axioms defining the head symbol of some (sub)term including the variables to be instantiated [5].

New reasoning techniques have been added to deal with non-trivial applications. The implementation of a combination between a decision procedure for linear arithmetic and a congruence closure algorithm [3], [35] allowed to validate the MJRTY algorithm [19] using a lemma proposed by N. Shankar (according to [27]); also, more than $60 \%$ of the conjectures required to certify the conformity algorithm for a telecommunication protocol [34] have been automatically proved. This implementation, as well as the 'black-box' integration schema of the Z3 [22] SMT solver as a reasoning module, are described in [39]. SPIKE also includes several decision procedures for proving inductive theorems without induction reasoning [2]. They help to decide the inductive validity of equations involving natural numbers and lists.

The validation of the JavaCard platform [5] was the most challenging case study ever experienced by SPIKE. The inference system has been adapted to manage variables of parameterized sorts as well as existential variables. New inference rules have been designed to better handle the information from the conditional part of (conditional) conjectures, like i) the auto simplification rule in order to rewrite with an equality condition other parts of the conjecture, and ii) the augmentation rule which adds new conditions issued from the conclusion of a conditional equality given as lemma if the conditions of the lemma are proved from the conditions of the conjecture. The efficiency of the implementation has been improved for dealing with specifications counting more than 400 defined function symbols and 2200 axioms, for example by recording the failure context at the conjecture level in order to avoid useless computation.

As shown in [21], the implicit induction method is based on a unique induction ordering, globally defined over the set of clauses derived during a proof. It is implemented by reductive inference systems such that, at the ground level, the new conjectures from any proof step are smaller than (and sometimes equal to) the replaced conjecture. The reductive techniques, as rewriting, introduce new ordering constraints to be satisfied by the specification as well as the conjectures from the proof derivation. The induction ordering is the multiset extension of the mpo ordering [4] over terms using a precedence over the function symbols provided by the user. The mpo ordering also serves to orient the axioms into rewrite rules. Since some reasoning techniques, like the instantiation of variables from the current conjecture, require the reduction of the instances by rewriting, SPIKE warns the user if the mpo ordering built from the input precedence cannot orient the axioms into rewrite rules. If no precedence is provided by the user, SPIKE analyses the axioms and tries to infer a successful precedence.

The inference system of SPIKE has been extended to implement for the first time reductive-free cyclic proofs [38], by keeping the best features of explicit and implicit induction reasoning. A cycle consists of a circular linked list of proof derivation chunks, called history chunks. Each link symbolises the application of an instance of the head conjecture from a 
history chunk as $\mathrm{IH}$ in the proof of the conjecture ending the previous history chunk in the list. By following a particular proof strategy, referred to as the $\mathrm{DRaCuLa}$ strategy, the cycle can discharge its linking IHs by checking ordering constraints involving only instances of the conjectures starting the history chunks. Therefore, the cyclic induction reasoning allows to use non-reductive proof techniques along history chunks and axioms not orientable into rewrite rules as long as the ordering constraints are satisfied.

A useful property for an inference system is the refutational soundness which guarantees that, whenever a counterexample is detected at the current step, the initial conjectures are refuted. Very few inference rules implemented by SPIKE may add new counterexamples during the proof derivation, e.g., by the generalisation of existential into universal variables [5]. By attaching history information to each conjecture, the detected counterexamples can lead to particular ground instances of the initial conjectures that can be further checked for validity.

The refutational completeness is another useful property, satisfied by previous inference systems [11], [15]. Since the addition of a strategy language, this property is no longer guaranteed because one of the conditions to be satisfied is the fairness of the proof strategy. However, the user still can use some built-in strategies known to be refutationally complete. A classical proof strategy mainly privileges the inference rules that are instances of DELETE, then SiMPLIFY, and finally ADDPREMISE. We give as example the definition of the fullind strategy.

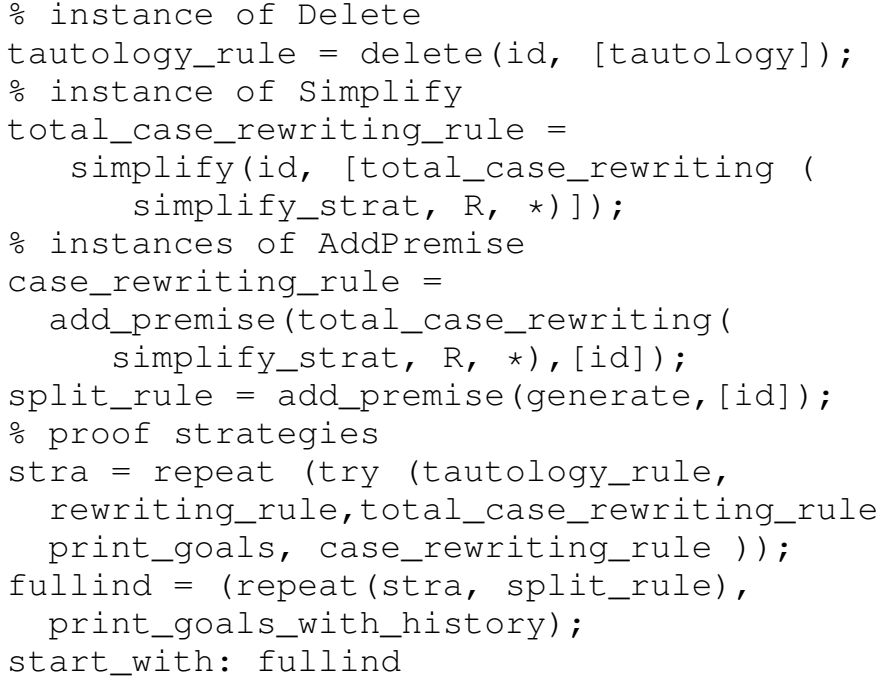

The reasoning module total_case_rewriting, implementing the conditional rewriting technique, was used to build instances of both SIMPLIFY and ADDPREMISE. try and repeat take a list of rules as arguments. try visits each rule in the list until the first succeeds. repeat executes them repeatedly until no new conjecture is produced or a counterexample is found. The print_* rules print the current state of the proof. start_with points to the used strategy.

c) Layout of a specification file: The structure of a standard SPIKE specification from the file name.spike is:

specification: name

sorts: list of sorts constructors: list of constructor symbols defined functions: list of defined function symbols axioms: list of axioms for each defined function symbol $\%$ the precedence used by the induction ordering greater: list of precedences over the function symbols equiv: list of equivalent function symbols

$\%$ the completeness and ground convergence properties properties: list of properties

$\%$ the proof strategies and conjectures strategy: list of inference rules and proof strategies conjectures: list of conjectures

d) User interactions: The proofs, generated by the command spike_bc name.spike, are highly automatic, the user interactions mainly defining $i$ ) the precedence used by the mpo ordering, ii) the inference rules and the proof strategy, iii) the precedence over the head symbols of the (sub)terms to which the new instantiation technique can be applied, and iv) lemmas. Once a conjecture has been proved, it can participate as lemma in the proof of further conjectures listed in the conjectures section.

On the other hand, the generated proofs may involve many non-trivial inference steps for which the human checking process is tedious and error-prone. We have shown how to i) validate SPIKE proofs [37], [41] using the certification environment provided by the Coq system [42], and ii) define Coq tactics that call directly SPIKE and transform the generated proofs into Coq scripts [26], according to a methodology that automatically translates into Coq script the proof steps performed by most of its inference rules [40].

The user can also interact with SPIKE by the means of i) extra sections, for example use: nats ${ }^{1}$ for activating the combination of the decision procedure for linear arithmetic and the congruence closure procedure, and ii) command-line arguments given to spike_bc, as:

-debug: to identify syntactic errors in the specification file,

-maximal: to print the proof in detail,

-coqc_spec and-coqc: to generate the Coq specification and translate the generated proof into Coq script, respectively, and

- dracula: to generate cyclic proofs using the DRaCuLa strategy.

3) Conclusions and Future Work: We have given an overview of the current version of SPIKE and highlighted the main changes and extensions since [14]. Initially written by Adel Bouhoula during his $\mathrm{PhD}$ thesis [8], SPIKE has been completely redesigned for adopting an object-oriented paradigm along the $\sim 18500$ lines of OCaml code. Some of the original features are still missing, like the graphical interface and the procedures for checking the completeness and ground convergence properties. In the future, an interesting application of SPIKE would be to the automation of the induction reasoning during the algorithm synthesis process [23]-[25].

Acknowledgments: The author would like to thank Adel Bouhoula and Michaël Rusinowitch, as well as the people involved in supporting, developing and using SPIKE.

${ }^{1}$ To be added just after the specification section. 


\section{REFERENCES}

[1] I. Alouini and A. Bouhoula. Un langage de stratégie pour SPIKE Working document, 1997.

[2] T. Aoto and S. Stratulat. Decision procedures for proving inductive theorems without induction. In Proc. of the 16th International Symposium on Principles and Practice of Declarative Programming (PPDP 2014), pages 237-248. ACM Press, 2014.

[3] A. Armando, M. Rusinowitch, and S. Stratulat. Incorporating decision procedures in implicit induction. Journal of Symbolic Computation, 34(4):241-258, 2002

[4] F. Baader and T. Nipkow. Term Rewriting and All That. Cambridge University Press, 1998.

[5] G. Barthe and S. Stratulat. Validation of the JavaCard platform with implicit induction techniques. In R. Nieuwenhuis, editor, RTA (Rewriting Techniques and Applications), volume 2706 of LNCS, pages 337-351. Springer, 2003.

[6] N. Berregeb, A. Bouhoula, and M. Rusinowitch. SPIKE-AC: A system for proofs by induction in associative-commutative theories. In H. Ganzinger, editor, Rewriting Techniques and Applications, volume 1103 of Lecture Notes in Computer Science, pages 428-431. Springer Berlin Heidelberg, 1996.

[7] N. Berregeb, A. Bouhoula, and M. Rusinowitch. Observational proofs with critical contexts. In Fundamental Approaches to Software Engineering, pages 38-53, 1998.

[8] A. Bouhoula. Preuves automatiques par récurrence dans les théories conditionnelles. PhD thesis, Université Henri Poincaré - Nancy I, 1994.

[9] A. Bouhoula. SPIKE: A system for sufficient completeness and parameterized inductive proofs. In A. Bundy, editor, Automated Deduction CADE-12, volume 814 of Lecture Notes in Computer Science, pages 836-840. Springer Berlin Heidelberg, 1994.

[10] A. Bouhoula. Using induction and rewriting to verify and complete parameterized specifications. Theoretical Computer Science, 170:170$1,1996$.

[11] A. Bouhoula. Automated theorem proving by test set induction. Journal of Symbolic Computation, 23(1):47-77, 1997.

[12] A. Bouhoula. Simultaneous checking of completeness and ground confluence for algebraic specifications. ACM Transactions on Computational Logic (TOCL), 10(3):1-33, 2009.

[13] A. Bouhoula and J.P. Jouannaud. Automata-Driven Automated Induction. Information and Computation, 169(1):1-22, 2001

[14] A. Bouhoula, E. Kounalis, and M. Rusinowitch. SPIKE, an automatic theorem prover. In Logic Programming and Automated Reasoning (LPAR), pages 460-462, 1992.

[15] A. Bouhoula, E. Kounalis, and M. Rusinowitch. Automated mathematical induction. Journal of Logic and Computation, 5(5):631-668, 1995

[16] A. Bouhoula and M. Rusinowitch. Automatic case analysis in proof by induction. In Proceedings of the 13th International Joint Conference on Artificial Intelligence (IJCAI), volume 1, pages 88-94, 1993.

[17] A. Bouhoula and M. Rusinowitch. Implicit induction in conditional theories. Journal of Automated Reasoning, 14(2):189-235, 1995.

[18] A. Bouhoula and M. Rusinowitch. Observational proofs by rewriting. Theoretical Computer Science, 275(1-2):675-698, 2002.

[19] R.S. Boyer and J S. Moore. MJRTY -A Fast Majority Vote Algorithm. Automated Reasoning: Essays in Honor of Woody Bledsoe, 1991.

[20] F. Bronsard and U. S. Reddy. Conditional rewriting in Focus. In Conditional and Typed Rewriting Systems, pages 1-13, 1991.

[21] F. Bronsard, U.S. Reddy, and R. Hasker. Induction using term orderings. In CADE (Conf. on Automated Deduction), volume 814 of $L N C S$, pages 102-117. Springer, 1994.

[22] L. De Moura and N. Bjørner. Z3: An efficient SMT solver. In Proceedings of the Theory and Practice of Software, 14th International Conference on Tools and Algorithms for the Construction and Analysis of Systems, TACAS'08/ETAPS'08, pages 337-340, Berlin, Heidelberg, 2008. Springer-Verlag.

[23] I. Dramnesc and T. Jebelean. Proof-based synthesis of sorting algorithms using multisets in Theorema. In M. Marin and A. Craciun, editors, Proceedings Third Symposium on Working Formal Methods,
FROM 2019, Timişoara, Romania, 3-5 September 2019, volume 303 of EPTCS, pages 76-91, 2019.

[24] I. Dramnesc and T. Jebelean. Synthesis of sorting algorithms using multisets in Theorema. Journal of Logical and Algebraic Methods in Programing, 2021. to appear. https://doi.org/10.1016/j.jlamp.2020.100635.

[25] I. Dramnesc, T. Jebelean, and S. Stratulat. Mechanical synthesis of sorting algorithms for binary trees by logic and combinatorial techniques. Journal of Symbolic Computation, 90:3-41, 2019.

[26] A. Henaien and S. Stratulat. Performing implicit induction reasoning with certifying proof environments. In A. Bouhoula, T. Ida, and F. Kamareddine, editors, Proceedings Fourth International Symposium on Symbolic Computation in Software Science, Gammarth, Tunisia, 15-17 December 2012, volume 122 of Electronic Proceedings in Theoretical Computer Science, pages 97-108. Open Publishing Association, 2013.

[27] D. J. Howe. Reasoning about functional programs in Nuprl. In Functional Programming, Concurrency, Simulation and Automated Reasoning, volume 693 of Lecture Notes in Computer Science, pages 145164. Springer Verlag, 1993

[28] G. Huet. The Gilbreath trick: A case study in axiomatisation and proof development in the Coq proof assistant. Technical Report RR-1511, INRIA, 1991.

[29] D. Kapur, P. Narendran, and H. Zhang. Proof by induction using test sets. In 8th International Conference on Automated Deduction, volume 230 of Lecture Notes Computer Science, pages 99-117. Springer, 1986.

[30] D. Kapur and H. Zhang. RRL: A rewrite rule laboratory. In Ewing Lusk and Ross Overbeek, editors, 9th International Conference on Automated Deduction, volume 310 of LNCS, pages 768-769. Springer Berlin / Heidelberg, 1988.

[31] E. Kounalis and M. Rusinowitch. Mechanizing inductive reasoning. In Proceedings of the eighth National conference on Artificial intelligence - Volume 1, AAAI'90, pages 240-245. AAAI Press, 1990.

[32] P. Lescanne. Implementation of completion by transition rules + control: ORME. In H. Kirchner and W. Wechler, editors, Algebraic and Logic Programming, volume 463 of Lecture Notes in Computer Science, pages 262-269. Springer Berlin Heidelberg, 1990.

[33] D. R. Musser. On proving inductive properties of abstract data types In $P O P L$, pages $154-162,1980$

[34] M. Rusinowitch, S. Stratulat, and F. Klay. Mechanical verification of an ideal incremental ABR conformance algorithm. Journal of Automated Reasoning, 30(2):153-177, 2003.

[35] S. Stratulat. Preuves par récurrence avec ensembles couvrants contextuels. Applications à la vérification de logiciels de télécommunications. PhD thesis, Université Henri Poincaré, Nancy I, November 2000.

[36] S. Stratulat. A general framework to build contextual cover set induction provers. Journal of Symbolic Computation, 32(4):403-445, 2001.

[37] S. Stratulat. Integrating implicit induction proofs into certified proof environments. In IFM'2010 (8th International Conference on Integrated Formal Methods), volume 6396 of Lecture Notes in Computer Science, pages 320-335, 2010

[38] S. Stratulat. A unified view of induction reasoning for first-order logic. In A. Voronkov, editor, Turing-100 (The Alan Turing Centenary Conference), volume 10 of EPiC Series, pages 326-352. EasyChair, 2012.

[39] S. Stratulat. Implementing reasoning modules in implicit induction theorem provers. In SYNASC (International Symposium on Symbolic and Numeric Algorithms for Scientific Computing), pages 133-140. IEEE Computer Society, 2014.

[40] S. Stratulat. Mechanically certifying formula-based Noetherian induction reasoning. Journal of Symbolic Computation, 80, Part 1:209-249, 2017.

[41] S. Stratulat and V. Demange. Automated certification of implicit induction proofs. In CPP'2011 (First International Conference on Certified Programs and Proofs), volume 7086 of Lecture Notes Computer Science, pages 37-53. Springer Verlag, 2011.

[42] The Coq development team. The Coq Reference Manual. INRIA, 2020. http://coq.inria.fr/doc.

[43] The SPIKE development team. The SPIKE prover, 2020. https://github. com/sorinica/spike-prover. 